\title{
An overview of COVID-19 vaccine candidates
}

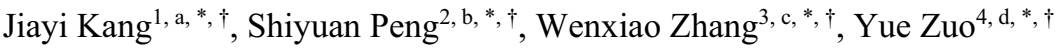 \\ ${ }^{1}$ Sargent College of Health and Rehabilitation Sciences, Boston University, Boston, MA 02215 \\ ${ }^{2}$ College of Life Science and Bioengineering, Beijing University of Technology, Beijing, China \\ ${ }^{3}$ School of Public Health, University of Washington, Seattle, WA 98105 \\ ${ }^{4}$ College of Agriculture and Life Sciences, North Carolina State University, Raleigh, NC 27695 \\ ${ }^{\dagger}$ These authors contributed equally.
}

\begin{abstract}
For the past two years, people worldwide were experiencing one of the most massive pandemics, the COVID-19. It is caused by a virus that is called Severe Acute Respiratory Syndrome Coronavirus 2 (SARS-CoV-2), which mainly attacks the respiratory system. Since there is no previous experience treating the disease, the drug development process is extremely difficult. People in dire need of vaccines were urgently needed to restore their lives to normal. As time went by, medical staff and experts spent numerous amounts of time and effort studying this virus. Nowadays, as the vaccine technology of various countries was very mature, vaccines with various technological paths have entered the experimental stage one after another. Eventually, effective vaccines are finally ready for wild inoculation for everyone by collaborating with all medical researchers. Currently, there are four kinds of COVID-19 vaccines available in different countries. They are inactivated vaccines, mRNA-based vaccines, viral vector vaccines, and recombinant vaccines. Most of these COVID-19 vaccines are in Phase 3 and provided in different countries. Those vaccines were manufactured in different countries, and their general production and technical characteristics will be discussed in the following paragraphs. The main purpose is trying to provide some fundamental and comprehensive background knowledge about currently available COVID-19 vaccines. It could be a reference list for those audiences who have not decided to take which vaccine.
\end{abstract}

\section{Introduction}

The novel coronavirus disease (COVID-19) outbreak, caused by respiratory syndrome coronavirus 2 (SARS$\mathrm{CoV}-2$ ), is seeing a rapid increase in infected patients worldwide. SARS-CoV-2 enters the human body by overcoming the species barrier and promotes person-toperson transmission. It has spread across 188 countries and 25 regions in the world since its emergence, even though the WHO and relevant governments have tried their best to constrain infection, which is mainly associated with its high infection[1]. Up to July 2nd, 2020 , there are $10,533,779$ global cases, including 512,842 death cases[2]. Besides, the infected cases have increased rapidly, and about 210,209 patients are diagnosed daily up to July 2nd, 2020.

Currently, it is urgent to develop an efficient vaccine for the prevention of future outbreaks and the control of this pandemic. In September 2020, Coalition for Epidemic Preparedness Innovations (CEPI), the multinational and multilateral stakeholders foundation founded to develop the anti-infectious disease vaccines, reported that 9 respective technological platforms are utilized for developing the efficient vaccine to resist SARS-CoV-2[3]. Moreover, scientists from the whole world have utilized the next-generation and classical platforms. Typically, next-generation platforms include viral vectors (replicating or non-replicating), nucleic acids (DNA or RNA), antigen-presenting cells, and recombinant proteins. In contrast, classical platforms include the live-attenuated virus whole-inactivated virus, virus-like particles, or protein subunit[4]. Up to November 11th, 2020, dose-confirmation, single-blind, double-blind, open-label non-randomized, and observerblind randomized studies have been proposed to prepare diverse vaccine types from different technological platforms, including 18 nucleic acids, 2 replicating and 8 non-replicating viral vectors, 11 protein subunit, 1 viruslike particle, 9 inactivated virus along with 3 additional candidate vaccines. From the perspective of clinical trials carried out, China has conducted the most clinical trials, higher than those conducted in other countries like the USA, Australia, Canada, and the UK. This review summarizes the technology, mechanism, and efficiency of four types of vaccine: inactivated vaccines, mRNAbased vaccine, recombinant vaccine, and viral vector vaccines.

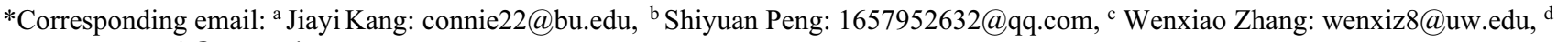
Yue Zuo: yzuo2@ncsu.edu. 


\section{An overview of the COVID-19 pandemic}

\subsection{Pandemic and vaccine administration}

The coronavirus disease 2019 (COVID-19) becomes one of the worst pandemics in history. It impacts many people around the world and "presents countries with major political, scientific, and public health challenges"[5]. The first case was reported in Wuhan, China, and the disease rapidly spread, resulting in an epidemic throughout China, followed by a global pandemic. According to the latest data, there are more than 130 million cases, and about 3 million died due to COVID-19 worldwide (Table 1).

Table 1. Statistics of COVID-19 (Retrieved 17 April 2021, from https://en.wikipedia.org/wiki/COVID-19_pandemic)

\begin{tabular}{lll}
\hline Location & Total Cases & Deaths \\
\hline Worldwide & $139,645,558$ & $2,996,305$ \\
United States & $139,645,558$ & 565,723 \\
India & $14,291,917$ & 174,308 \\
Brazil & $13,746,681$ & 365,444 \\
France & $5,149,776$ & 99,427 \\
Russia & $4,622,464$ & 102,667 \\
United Kingdom & $4,380,976$ & 127,191 \\
\hline
\end{tabular}

"Vaccines reduce risks of getting a disease by working with your body's natural defenses to build protection and are also critical to the prevention and control of infectious-disease outbreaks" [6]. In other words, vaccine administration provides a cure rather than a treatment to the COVID-19 pandemic. COVID-19 vaccines were developed quickly, and more than 300 million people are vaccinated around the world so far.

\subsection{SARS-CoV2 and mutations}

Coronavirus is a large family of viruses that cause mild to severe respiratory infections in humans. "In 2002 and 2012 , two highly pathogenic coronaviruses with zoonotic origin, severe acute respiratory syndrome coronavirus (SARS-CoV) and Middle East respiratory syndrome coronavirus (MERS-CoV), emerged in humans and caused fatal respiratory illness"[7]. Severe acute respiratory syndrome coronavirus 2 (SARS-CoV-2) is the newly discovered coronavirus that causes the infectious disease COVID-19. This disease is mainly spread by person-to-person transmission, and the most common symptoms are fever, coughing, and breathing problems. Most people can be treated at home to get cold or flu and recover without the need for hospital care. People who have severe symptoms need to get medical care and treatment in hospitals.
Several new SARS-CoV-2 variants are containing one or more mutations discovered and reported globally. B.1.526 and B.1.525 were two new variants first detected from the United States in 2020. P.2 was detected in Brazil in 2020. "These variants share one specific mutation called D614G," and the evidence shows that the variants with D614G could spread faster. B.1.1.7 detected from the United Kingdom and B.1.351 detected from South Africa have about 50\% increased transmission. These two variants share one specific mutation called D614G, which causes them to spread out rapidly and distribute globally. Besides, evidence shows that the new variants have a significant reduction in neutralization by antibodies generated during previous infection or vaccination[8]. The efficiency of vaccines and treatment are reduced for new SARS-CoV-2 variants.

\section{Inactivated vaccines}

\subsection{Technical Principles}

An inactivated vaccine is a vaccine composed of virus particles, bacteria, or other processed pathogens treated with proper techniques. The most important principle of making an inactivated vaccine is to ensure the cultured pathogen has been killed to destroy disease-producing capacity [9]. Some classic diseases are commonly treated by applying the inactivated vaccine. Those are Polio (IPV), Hepatitis A, and Rabies[10]. Once the inactivated virus enters the human body, the immune system will be triggered and make a series of responses to identify those invaders and remove them from the human body. The adaptive immune system will generate B cells and T cells to memorize a specific virus and be prepared to remind the immune system to secret antibodies in the future.

\subsection{Technical Characteristics}

There are different types of vaccines finally ready for people to get inoculated. One of the most common types of vaccine is the inactivated vaccine. It is a vaccine composed of dead or inactivated viruses and bacteria, which is different from live but attenuated vaccines. Inactivated vaccines will not provide strong reactions within the human body compared to live vaccines, which means this vaccine can be administered when antibodies are present in the bloodstream[11]. Inactivated vaccines are unable to replicate in the host body. Another benefit of the inactivated vaccine is that it can be used as a boost to supplement previous vaccinations since inactivated vaccines only show rare or no cellular immunity. Last but not least, inactivated vaccines can be frozen, which means they can be stored easily. Moreover, an inactivated vaccine will not experience mutation since all viruses were killed during the production[11].

\subsection{CoronaVac}

CoronaVac is an inactivated vaccine produced by Sinovac, which has been in Phase III clinical trials in 
different countries, such as Brazil, Chile, Indonesia, Malaysia, Philippines, and Turkey[12]. CoronaVac is usually inoculated with aluminum as an adjuvant. With the help of aluminum salts, a strong enough immune response will be produced to protect individuals from various diseases caused by vaccination. In this case, adjuvants can increase the safety and effectiveness of the vaccine. Some local reactions caused by adjuvants are redness, swelling, and pain at the injection site (CDC)[13]. Based on the experiments, 3 and $6 \mu \mathrm{g}$ are the ideal doses since they will lead to high NAb (neutralizing antibody) responses, which defend a cell from a pathogen or infection by neutralizing them. The level of $\mathrm{NAb}$ for the first dose is relatively stable within 0-14 days. Moreover, the level of NAb is considerably increased after the second dose. In this case, the most ideal and effective inoculation plan for CoronaVac is a 3 $\mu \mathrm{g}$ dose in 0-, 14-day, and 0-, 28-day schedules[12]. The current efficacy of CoronaVac is between $50.65 \%$ and $83.5 \%$, based on the trials from Brazil, Turkey, and Indonesia[14].

\subsection{BBIBP-CorV}

BBIBP-CorV is an inactivated vaccine that was produced by Sinopharm and initiated a Phase III study in different countries all over the world. For BBIBP-CorV[12]. Vero cells are applied during production. Vero cells are a lineage of cell that is most commonly used in cell cultures. According to researchers, this linkage was isolated from kidney epithelial cells extracted from an African green monkey. The SARS-CoV-2 was cultivated in a qualified Vero cell line for propagation. Moreover, $\beta$-propiolactone is adopted to inactivate the supernatant of infected cells[15]. This vaccine also utilizes adjuvants for better inoculation, based on the formula, sodium salts, and aluminum salts are added to. The specification of BBIBP-CorV is provided in the instruction, each dose for single use is $0.5 \mathrm{~mL}$, and each dose contains $4 \mu \mathrm{g}$ of inactivated SARS-CoV-2 protein. Two doses are recommended, and the interval between each dose is approximately 2 to 4 weeks[16]. According to the results, ADE (antibody-dependent enhancement) is not introduced during the current experiment, and a highly effective immune response appears in different sizes of doses. The CNBG (China National Biotec Group) announced that the vaccine was $79.34 \%$ effective on the prevention based on the interim data[14].

\section{Recombinant vaccines}

Besides inactivated and live attenuated, the recombinant vaccine is a widely developed vaccine for many diseases. It includes protein-based vaccines, virus-like particlebased vaccines, nucleic acid-based coronavirus vaccines, and vector-based coronavirus vaccines. This vaccine uses specific pieces of such as protein. Compared to the other vaccines, the recombinant vaccine could "give a very strong immune response that's targeted to key parts of the germ"[17]. It is usually used for people with weakened immune systems or long-term health problems like HIV and Hepatitis B.

\section{1 mRNA-based vaccines}

\subsubsection{Introduction of $m R N A$-based vaccines}

mRNA vaccines are the novel nucleic acid vaccine type based on their higher protein levels and less mutagenesis than DNA vaccines. Numerous target proteins are expressed within immune cells when mRNA vaccines are used, thus exerting effective immunization. To take an example, antigen-specific vaccination may be adopted, where specific antigens are expressed within the antigenpresenting cells. Immune responses can be regulated through the expression of certain proteins related to immune cell maturation or stimulation. Besides, mRNA vaccines may also trigger innate immunity by the pattern recognition receptors-mediated specific recognition. Considering the above favorable characteristics, mRNA vaccines are applied in prophylactics and treatment.

Messenger RNA (mRNA) vaccines have evolved as a new type of nucleic acid vaccine according to their superior protein expression and a lack of mutagenesis compared with DNA vaccines. Using mRNA vaccines, large amounts of target proteins can be expressed in immune cells for efficient immunization. For instance, antigen-specific vaccination is a feasible option involving the expression of specific antigens in antigenpresenting cells. Immunological reactions are modulated by expressing several proteins associated with the stimulation or maturation of immune cells. In addition, mRNA vaccines can stimulate innate immunity through specific recognition by pattern recognition receptors. MRNA vaccines have been used for prophylactic and therapeutic applications[18].

The mRNA produced in the laboratory is used in mRNA vaccines after being wrapped in nanoparticles (NPs). mRNA translation promotes the protein antigen development, which can promote the immune reaction. mRNA vaccines contribute to the direct delivery of mRNA into the cytoplasm, and then mRNA will be transcribed via ribosomes in the cytoplasm. mRNA is not transported into the nucleus. As a result, it is not included in the genome. It exists transiently in cells since it will be rapidly metabolized and removed by the cell processing mechanisms. No organism-derived element is utilized in mRNA vaccines[19].

Notably, mRNA-based vaccines are associated with 3 advantages. Firstly, they are non-infectious since they are not prepared using inactivated pathogens or pathogen particles. RNA will not be integrated into the host genome; besides, after producing a protein, RNA strands within the vaccines will be decomposed. Secondly, as revealed by previous clinical results, mRNA vaccines produce the creditable immune reaction, which shows good tolerance among the normal subjects, and a low adverse reaction rate is reported. Thirdly, laboratory vaccines are manufactured rapidly by the standardized process, thus improving their responsiveness to outbreaks of emerging diseases. 


\subsubsection{Mechanism of mRNA-based vaccines}

mRNA, the single-stranded RNA molecule that complements DNA, is contained in mRNA vaccines, which can be synthesized within the cell nucleus once RNA polymerase transcribes DNA to produce premRNA. After that, pre-mRNA can be spliced to mRNA, followed by cytoplasmic transport from the cell nucleus and "read" via ribosomes (cell translation mechanism). Later, ribosomes produce corresponding proteins.

mRNA vaccines, which function based on the expression of ORF viral sequences-containing chimeric mRNAs in the cytoplasm, may be potentially directly translated within the cytoplasm, thus blocking the integration of chromosomes. After injection, immune cells will process the mRNA delivered and generate targeted protein through translation; afterward, additional immune cells will be activated to recognize the new viral proteins generated, thus generating specific antibodies[20].

There are 2 kinds of RNA vaccines developed to resist infectious pathogens: replicon or self-amplifying RNA vaccine and non-replicating mRNA vaccine. Because of the diverse delivery modes, non-replicating mRNA vaccines can be additionally classified into direct in vivo injection to different anatomical positions and dendritic cell loading ex vivo. To arrive at cytoplasm, exogenous mRNA should penetrate the lipid membrane barrier before it is translated into different proteins. Additionally, the mRNA vaccine uptake mechanism is cell-specific; meanwhile, mRNA physicochemical characteristics significantly impact the organ distribution and cell delivery of mRNA. The above factors should be taken into consideration in the design of efficient mRNA-based vaccines. Such as it is, mRNA vaccines are recognized as the potential candidates' thanks to their rapid scaling. This is time-saving in the presence of the rapid COVID-19 emergence and transmission among all populations in the world[20].

SARS-CoV-2 is a kind of (+)ss-RNA virus possessing the self-amplifying RNA to allow cytosolic RNA replication. This finding provides support for the role of mRNA-based vaccine development. Nevertheless, the safety and efficacy of mRNA vaccines for use in humans remain unclear. mRNA vaccines appear to possess great putative advantages. Still, they are also associated with certain limitations, like RNA degradation-related stability and delivery problems and immunogenicity-related safety concerns, which have hindered their development.

\subsubsection{Moderna mRNA vaccines}

At present, the candidate mRNA-1273 vaccine produced by Moderna can encode S-2P antigen, which comprises SARS-CoV-2 glycoprotein possessing an intact S1-S2 cleavage site and a transmembrane anchor. S-2P can be stabilized into the prefusion conformation via 2 successive proline substitutions at 986 and 987 positions on top of the S2 subunit central helix. The lipid NP capsule containing 4 lipids will be developed at the constant mRNA-to-lipid ratio. The mRNA-1273 vaccine was provided as a sterile liquid for injection at a concentration of $0.5 \mathrm{mg}$ per milliliter. Normal saline was employed as a diluent to make the preparation for the doses administered[21].

\subsection{Protein-based coronavirus vaccine}

\subsubsection{Introduction of protein-based coronavirus vaccine}

Protein-based coronavirus vaccine is one the most frequently mentioned kinds of recombinant vaccine. "The $\mathrm{S}$ protein is a good candidate target for recombinant vaccine development because SARS-CoV-2 vaccine based on the $\mathrm{S}$ protein may efficiently induce the production of antibodies and virus neutralization"[22]. The protein-based COVID-19 recombinant vaccine uses recombinant S-protein to mimic SARS-Cov-2 $\mathrm{S}$ protein to get $\mathrm{S}$ protein expression inside the host body. Vaccinated people will have specific antibodies against SARS-Cov-2 S protein, and the immune response leads to immunization.

\subsubsection{Current vaccine candidates in clinical trials}

According to the data of COVID-19 candidate vaccines in clinical trials from WHO, more than three of proteinbased COVID-19 recombinant vaccines are in phase 1 in clinical trials. $2019 \mathrm{nCoV}-101$ is a phase 2 clinical trial sponsored by Novavax. It is the "evaluation of the safety and immunogenicity of a SARS-Cov-2 $\mathrm{rS}$ nanoparticle vaccine with/without matrix-m adjuvant" (ClinicalTrials.gov Identifier: NCT04368988) and will complete November 18, 2021. Also, on Jan. 28, 2021, Novavax announced that "NVX-CoV2373, its proteinbased COVID-19 vaccine candidate, demonstrates $89.3 \%$ Efficacy in the United Kingdom (UK)UK Phase 3 Trial"[23]. What the good news is this vaccine has high clinical efficacy against UK and South Africa variants. Many other clinical trials of protein-based COVID-19 recombinant vaccine development in the process also show that recombinant vaccines may be efficient against SARS-Cov-2.

\subsection{Viral vector vaccines}

A viral vector vaccine is a novel vaccine that consists of a modified version of one virus as a vector with an antigen or antigen's DNA sequence for another infectious agent inside. Before the viral vector vaccine for COVID-19, it was only authorized for the ebola virus[24]. In the past few years, scientists have always sought solutions from this technology for virus infection, like the zika virus, influenza viruses, HIV, and malaria. Apart from poxvirus and vesicular stomatitis virus vector for ebolavirus vaccine, by April 2021, all four viral vector vaccines for COVID-19 are based on adenovirus. 


\subsubsection{Adenoviruses}

Until now, adenoviruses are the most popular and advanced viral vectors for the novel coronavirus. DNA viruses without envelope protein can package up to 7.5 $\mathrm{kb}$ of foreign genes[25]. Relying on the specially designed vector can ensure both humoral and cytotoxic (CD8+) T cell responses are induced efficiently, which shows obvious advantages over subunit vaccines. More, viral vectors can also act as adjuvant due to their diverse pathogen-associated molecular patterns, which activate innate immunity. Some replication-competent viral vectors can provide equivalent potency at lower doses compared to other types of vaccines[26]. However, all four authorized viral vector vaccines are nonreplicating[27].

Viral vector vaccines also have drawbacks: preexisting immunity. It may slow down the rapid immune response to sars-cov-2 and reduce the peak of the response and persistence[28]. It can be overcome with higher doses of usage, tolerability permitting, and heterologous prime-boost vaccine regimens'[26].

\subsubsection{Developing and applying condition of viral vector vaccines}

According to WHO's draft landscape and tracker of COVID-19 candidate vaccines, until April 9th, there is a total of 16 viral vector vaccines in the clinical phase, which takes up $19 \%$ of all types of vaccines in the clinical phase. Among those, four different types of vectors are used: Ad5, Ad26, ChAd, VSV. Six vaccines have their EOI(Expression Of Interest) accepted by the WHO.

By April 2021, there are four viral vector vaccines approved by at least one country. They are Ad5nCoV(Convidicea) from CanSino, Sputnik V from Gamaleya, Ad26.COV2.S from Johnson \& Johnson and AZD1222 from Oxford/AstraZeneca. The following part will compare and contrast the characteristics of the four vaccines.

For vector types: Sputnik V uses Ad5 and Ad26 vector, Convidicea uses only $\mathrm{Ad} 5$ as a viral vector, Ad26.COV2.S uses Ad26 as a viral vector, and AZD1222 uses the ChAd as the vector. Specifically, Convidicea is made by 'an optimised full-length spike gene with the tissue plasminogen activator signal peptide gene into an E1, and E3 deleted Ad5 vector'[29]. It is developed with the same platform for the Ebola vaccine, approved by the Chinese regulators in 2017 for the Ebola vaccine[30]. Although convinced has been approved for its good tolerance and immunogenicity, Ad5 still has its inherent drawback of pre-existing immunity against the Ad5 vector itself. The phase I clinical trial of Convidicea also confirmed that individuals with high pre-existing Ad5 immunity show lower vaccine efficiency[29, 31]. For Ad26 in Ad26.COV2.S, this situation is even worse: the seroprevalence of $\operatorname{Ad} 26$ reaches almost $40 \%$ in humans[32, 33]. ChAd is not a human adenovirus, so vaccines from Oxford/AstraZeneca have less worry about this[34]. Both vaccines from Oxford/AstraZeneca and Gamaleya need two shots with three-to-four-week intervals, and vaccines from CanSino and Johnson \& Johnson only require one shot. All four vaccines can store temporarily between two centigrade to eight centigrade.

During the clinical trial phases I and II, all four vaccines are shown to be safe and immunogenic. These antibodies induced against the spick protein and a measurable cellular immune response can both be detected[27]. The vaccine efficacy of the four vaccines is $66.3 \%$ and $85 \%$ for severe illness (Johnson \& Johnson), $90 \%$ for severe illness (CanSino), $76.0 \%$ (a single standard dose from day 22 to day 90) (Oxford/AstraZeneca) and 91.6\% (21 days after the first dose) (Sputnik V). Although the efficacy may differ with different individuals under specific circumstances, all four vaccines are still competent to give enough protection. Now, all four vaccines are experiencing the Randomized, Double-blind, Placebo-controlled Phase 3 clinical trials. Because the estimated study completion date remains several months, we do not know the results yet (NCT04505722, NCT04530396, NCT04526990, NCT04516746).

\subsubsection{Ethical consideration of viral vector vaccines}

In March, more than 20 countries have announced that they have paused the administration of vaccines from Oxford/AstraZeneca. According to the latest research from Paul Ehrlich Institute, at least 7 types of blood clots have a relationship with the administration of the vaccine from Oxford/AstraZeneca. Among those, the formation of three types of blood clots will endanger the life of the vaccinator. Previous studies also showed that viral vectors have a firm relationship with thrombocytopenia with possible mechanisms of binding with CAR receptor, binding with integrin receptor, or reacting with PAF receptor on platelet cell[35]. Although the benefits of vaccination significantly outweigh the risks, especially for those with a high risk of infection or with weak immune systems, the ethical issues of those fastdeveloped vaccines should attach great importance from and after this incident. Any newly developed vaccines always have the possible existence of risks that have not been identified yet. Only with enough diverse and longlasting clinical trials can we determine if some problems that only show in the later phases of clinical trials exist[36].

\section{Conclusion}

COVID-19 vaccines are the only effective treatment to prevent the infection of SARS-CoV-2 and control the COVID-19 outbreak. These vaccines were developed quickly, and more than 300 million people are vaccinated around the world so far. The two kinds of vaccines introduced above, inactivated vaccines and recombinant vaccines, are widely used in scientific research, clinical research, and immunization. "An inactivated vaccine is a vaccine that is composed of virus particles, bacteria or other processed pathogens that have been treated with proper techniques." Many inactivated vaccines are in 
Phase 3 clinical trials. "A recombinant vaccine is a vaccine produced through recombinant DNA technology. "There are three species of recombinant COVID-19 vaccines, mRNA-based vaccines, protein-based vaccines, and viral vector vaccines. The vaccine is a critical approach to treat and control the further spread of COVID-19. As the technology of the COVID-19 vaccine continues to mature, it is essential and important to understand how this newly developed vaccine works in the human body, which will help you find the most appropriate vaccine for inoculation. Hopefully, herd immunity will gradually be achieved by the mutual effort of people all around the world. I believe that we will conquer the COVID-19 one day in the future.

\section{References}

1. UW-Madison, FluGen, Bharat Biotech to develop CoroFlu, a coronavirus vaccine | Business Wire. 2021.

2. Coronavirus disease (COVID-19): situation report, 164. 2021.

3. Gopinathan, U., et al., R\&D for Emerging Infectious Diseases of Epidemic Potential: Sharing Risks and Benefits Through a New Coalition. 2020: Infectious Diseases in the New Millennium.

4. van Riel, D. and E. de Wit, Next-generation vaccine platforms for COVID-19. Nat Mater, 2020. 19(8): p. 810-812.

5. McCloskey, B., et al., Mass gathering events and reducing further global spread of COVID-19: a political and public health dilemma. Lancet, 2020. 395(10230): p. 1096-1099.

6. Vaccines and immunization. 2021.

7. $\mathrm{Hu}, \mathrm{B}$., et al., Characteristics of SARS-CoV-2 and COVID-19. Nat Rev Microbiol, 2021. 19(3): p. 141154.

8. SARS-CoV-2 Variants of Concern|CDC. 2021.

9. Inactivated vaccine - Wikipedia. 2021.

10. Vaccine Types|HHS.gov. 2021.

11. What is an Inactivated Vaccine?. 2021.

12. Sharma, O., et al., A Review of the Progress and Challenges of Developing a Vaccine for COVID-19. Front Immunol, 2020. 11: p. 585354.

13. Adjuvants and Vaccines | Vaccine Safety | CDC. 2021.

14. Sinopharm, Sinovac COVID-19 vaccine data show efficacy: WHO | Reuters. 2021.

15. Sinopharm's COVID-19 Vaccine Shows 86\% Efficacy, UAE Health Agency Says | BioSpace. 2021.

16. Full list of adverse reactions from China's Sinopharm vaccine revealed | Taiwan News 2021/01/11. 2021.

17. Vaccine Basics | HHS.gov. 2021.
18. Kyuri, L., et al., Development of mRNA vaccines and their prophylactic and therapeutic applications. Nano Research, 2018. 11: p. 5173-5192.

19. SARS-CoV-2 mRNA vaccine design enabled by prototype pathogen preparedness. Nature.

20. Meo, S.A., et al., COVID-19 vaccines: comparison of biological, pharmacological characteristics and adverse effects of Pfizer/BioNTech and Moderna Vaccines. 2021.

21. Jackson, L.A., et al., An mRNA vaccine against SARS-COV-2 - Preliminary report. New England Journal of Medicine, 2020. 383(20).

22. Yadav, T., et al., Recombinant vaccines for COVID19. Human Vaccines \& Immunotherapeutics, 2020. 16(12): p. 2905-2912.

23. Novavax COVID-19 Vaccine Demonstrates $89.3 \%$ Efficacy in UK Phase 3 Trial | Novavax Inc. - IR Site. 2021.

24. Ewer, K.J., et al., Viral vectors as vaccine platforms: from immunogenicity to impact. Curr Opin Immunol, 2016. 41: p. 47-54.

25. Schiedner, G., et al., Genomic DNA transfer with a high-capacity adenovirus vector results in improved in vivo gene expression and decreased toxicity. Nat Genet, 1998. 18(2): p. 180-3.

26. Ewer, K.J., et al., Viral vectors as vaccine platforms: from immunogenicity to impact. Curr Opin Immunol, 2016. 41: p. 47-54.

27. Izda, V., M.A. Jeffries and A.H. Sawalha, COVID19: A review of therapeutic strategies and vaccine candidates. Clin Immunol, 2021. 222: p. 108634.

28. Wei, C. and Hao, Q., Academician Wei Chen's COVID-19 vaccine clinical trial results released: all 108 volunteers developed an immune response. Science Grand View Garden, 2020(11): page 4.

29. Zhu, F.C., et al., Safety, tolerability, and immunogenicity of a recombinant adenovirus type-5 vectored COVID-19 vaccine: a dose-escalation, open-label, non-randomised, first-in-human trial. Lancet, 2020. 395(10240): p. 1845-1854.

30. Soleimanpour, S. and A. Yaghoubi, COVID-19 vaccine: where are we now and where should we go? Expert Rev Vaccines, 2021. 20(1): p. 23-44.

31. Ura, T., et al., New vaccine production platforms used in developing SARS-CoV-2 vaccine candidates. Vaccine, 2021. 39(2): p. 197-201.

32. Colloca, S., et al., Vaccine vectors derived from a large collection of simian adenoviruses induce potent cellular immunity across multiple species. Sci Transl Med, 2012. 4(115): p. 115ra2.

33. Shahcheraghi, S.H., et al., An overview of vaccine development for COVID-19. Ther Deliv, 2021. 12(3): p. 235-244.

34. Ura, T., K. Okuda and M. Shimada, Developments in Viral Vector-Based Vaccines. Vaccines (Basel), 2014. 2(3): p. 624-41. 
35. Yu, X., Xin, X. and Jin, Y., Research progress of type 5 adenovirus vector and thrombocytopenia. Journal of Modern Laboratory Medicine, 2011. 26(5): pp. 160-161.

36. Wibawa, T., COVID-19 vaccine research and development: ethical issues. Trop Med Int Health, 2021. 26(1): p. 14-19. 